\title{
Cobertura de la atención de la enfermedad que causa la muerte y lugar de ocurrencia del deceso, en Chile y la sexta región, 1990-2003
}

\author{
Heidy Leiva $\mathbf{H}^{1}$, Francisco León $\mathrm{K}^{2}$. \\ Medical coverage of the disease that \\ causes death and place of death in the \\ sixth region of Chile from 1990 to 2003
}

Background: The place of death has changed and coverage of medical attention at death varies according to the development level of countries. Aim: To assess the evolution of coverage of medical attention of the last disease and place of death as indicators of health care quality and development level, in the sixth region of Chile. Material and methods: We analyzed the Chilean death certificate data bases for the period 1990-2003. We performed linear regression analysis of death year with place of death and medical attention at death, comparing the sixth region and the rest of Chile. Results: The population under study corresponds to 1,102,896 death records, from 1990 to 2003. Proportion of deaths at home decreased in sixth region and increased in the rest of our country. Deaths at home for individuals under 15 years of age, decreased. Deaths in places different from home or hospital increased progressively in elderly people. The same happened among adolescents, probably related to the high proportion of deaths due to trauma. Conclusions: Coverage of attention of diseases that cause death improved. Place of death varies according to age. There is a remarkable increase in the access of children to hospitals, for the treatment of severe diseases. These results indicate that Chilean healthcare system quality has improved (Rev Méd Chile 2007; 135: 1025-33).

(Key w ords: Death; Delivery of health care; Health Services accessibility)

Recibido el 11 de octubre, 2006. Aceptado el 1 de marzo, 2007.

${ }^{1}$ Servicio de Pediatría, Hospital Regional de Rancagua, Chile. ${ }^{2}$ Servicio de Pediatría, Hospital de San Fernando, Chile.

T a evolución de la mortalidad en los países ـpresenta diferencias y es así como algunos países en vías de desarrollo, presentan estancamiento e incluso empeoramiento de las tasas de

Correspondencia a: Heidy Leiva. Alameda $6116^{\underline{0}}$ piso, Rancagua. Fax: 72234803. E mail: draheidy@terra.cl mortalidad $^{1}$. Otros, como Rusia, muestran que la mortalidad infantil tiende a descender pero, la esperanza de vida ha disminuido, prueba de la influencia del empobrecimiento de la población ${ }^{2}$. Hay evidencias de la importancia del género en la mortalidad y esperanza de vida, apareciendo las mujeres con una situación ventajosa ${ }^{2,3}$. Los cambios en la mortalidad tienen múltiples causas, 
destacando intervenciones sanitarias, factores sociales como el estado civil y existencia de familia ${ }^{4-6}$. La mortalidad general y especialmente la mortalidad infantil, han disminuido en el período 19902003, en Chile y en la Sexta Región, demostrando mejoramiento del nivel de salud de la población? ${ }^{7}$. En Chile existe registro confiable de las defunciones y la información obtenida desde el certificado de defunción permite concluir certeramente ${ }^{8}$.

El lugar en que se produce el deceso ha cambiado a lo largo de los años y hay diferencias por grupos etarios. En Chile, en un estudio sobre mortalidad por infarto agudo del miocardio, se constata que $43,9 \%$ de los pacientes varones y $43,6 \%$ de las mujeres fallecieron en un hospital, el año 1998; situación que vanía el año 2001 , en que $42,1 \%$ de los hombres y $42,5 \%$ de las mujeres mueren en un hospital, cobrando mayor importancia otros lugares de deceso $(4,9 \%$ en 1998 y $7 \%$ en 2001 para los casos de infartos en mujeres) ${ }^{9}$. En Colombia, en el quinquenio 1970-1974, 53,1\% de los pacientes fallecidos por infarto agudo de miocardio fallecen en el hogar, mientras que en el peńodo 20002002, 47,6\% de las muertes ocurren en la casa, aumentando el deceso en hospitales a $43,9 \%{ }^{10}$. Estudios alemanes demuestran que más de $50 \%$ de los pacientes terminales prefieren morir en su casa y que aquellos ancianos que padecen demencia tienen menos posibilidades de ser ingresados en un hospital y hay una mayor proporción de muertes en residencias para adultos mayores 0 en sus hogares ${ }^{11}$. En Suecia, Westerling demostró que una alta proponción de las muertes ocurren fuera del hospital. En India, $22 \%$ a $30 \%$ de los decesos ocurren en hospitales, mientras que $68 \%$ se produce en la casa y sin una clara definición de la causa del fallecimiento ${ }^{12}$. En los Estados Unidos de Norteamérica (EE.UU.), la casa es desplazada como sitio de ocurrencia de la muerte por instituciones médicas y actualmente, más norteamencanos mueren en hospitales ${ }^{13}$. Sin embargo, existen diferencias según las etnias de los pacientes ${ }^{14}$.

La cobertura de atención médica de enfermedad que causa la muerte, es diferente según el nivel de desarrollo. En Chile, en las muertes por infarto agudo de miocardio se aprecia mejoría de la cobertura y es así como en 1998, 23,4\% de los hombres y $16,7 \%$ de las mujeres no recibieron atención profesional, mientras que en 2001 estas cifras correspondieron a $21,3 \%$ y $15,7 \%$, respectivamente $^{9}$. En India, existe alta proporción de muertes sin atención médica, situación 11 veces mayor en las zonas rurales de ese país ${ }^{12}$. China ha implementado un sistema cooperativo de asistencia médica para enfrentar las enfermedades graves $^{4}$. En contraste, en EE.UU. aproximadamente un cuarto de los enfermos reciben tratamiento intensivo antes de fallecer ${ }^{13}$. En Francia, se ha hecho un esfuerzo especial en la atención de los pacientes terminales y existen experiencias de equipos móviles de cuidados paliativos que aseguran que, aunque el deceso se produzca en el hogar, sea asistido en forma profesional ${ }^{15}$. En el grupo de adolescentes, las causas de muertes están relacionadas con traumatismos y envenenamientos y en consecuencia una proporción importante de los decesos son fuera del hospital ${ }^{16-18}$.

Nuestro interés se ha centrado en el análisis de estos dos aspectos sanitarios, la cobertura de la atención médica de la muerte y el lugar de defunción, para lo que hemos revisado los antecedentes de las defunciones del período 1990-2003 de Chile y de nuestra región. La cobertura de la atención es considerada como un indicador de calidad del sistema de atención de salud y, en consecuencia, debe mejorar a lo largo de los años. En cambio, el lugar de defunción es variable dependiendo del grupo etario, de la cultura social en relación a la muerte y de las políticas de salud respecto de la disponibilidad de cuidados en la casa para el grupo de edad mayor. El propósito de este estudio fue evaluar la calidad de la atención del sistema de salud chileno. Los objetivos específicos fueron conocer la evolución de la cobertura de la atención de la enfermedad que causó la muerte y del lugar en que ocurrió el deceso y comparar la situación de los parámetros descritos en la Sexta Región con el resto del país.

\section{MAterial y MÉTOdo}

Analizamos la base de datos de certificados de defunción de Chile de 1990 a 2003, disponible en el sitio web del Departamento de Estadísticas e Información de Salud del Ministerio de Salud de Chile. Las variables estudiadas fueron año de defunción, edad, sexo, lugar de residencia, local de defunción y atención médica de última enfermedad. Se crearon las variables grupo etario: grupo infantil (menores de 15 años), grupo adolescente (15 a menores de 19 años), grupo adulto (19 a menores de 65 años) y 
grupo senescente (65 o más años); y la variable geográfica, clasificando en 2 grupos, defunciones de personas que residían en la Sexta Región y el resto del país. Se utilizó el programa estadístico SPSS 12.0, realizando análisis de regresión lineal, correlación y test t para muestras pareadas.

\section{RESULTADOS}

La población en estudio correspondió a 1.102.896 defunciones, 1990-2003 (Tabla 1).

El 54,8\% de las muertes correspondió a varones. Se excluyó a 0,1\% de los datos por carecer de información precisa del lugar de defunción.

$\mathrm{Si}$ observamos la evolución de las variables en el periodo 1990-2003, podemos señalar que: la proporción de defunciones en el hospital disminuyó en el resto del país $(R=0,44, p=0,496)$ y aumentó en la Sexta Región ( $R=0,311, p=0,28$ ), sin significación estadística. La Sexta Región mostró un promedio de muerte en hospital mayor que el resto de Chile ( $\mathrm{p}=0,012)$. En contraste, la proporción de muertes en casa aumentó en el resto del país y disminuyó en la región ( $R=0,643, p<0,0001)$. Sin embargo, la proporción de muertes en la casa fue mayor en la Sexta Región ( $p<0,0001$ ) (Tabla 2).
Tabla 1. D efunciones en Chile: 1990-2003

\begin{tabular}{|lrr|}
\hline Año & № & $\%$ \\
\hline 1990 & 78.434 & 7,1 \\
1991 & 74.862 & 6,8 \\
1992 & 74.090 & 6,7 \\
1993 & 76.261 & 6,9 \\
1994 & 75.445 & 6,8 \\
1995 & 78.531 & 7,1 \\
1996 & 79.123 & 7,2 \\
1997 & 78.472 & 7,1 \\
1998 & 80.257 & 7,3 \\
1999 & 81.984 & 7,4 \\
2000 & 78.814 & 7,1 \\
2001 & 81.871 & 7,4 \\
2002 & 81.080 & 7,4 \\
2003 & 83.672 & 7,6 \\
Total & 1.102 .896 & 100,0 \\
\hline
\end{tabular}

Defunciones de menores de 15 años 1990-2003 (Tabla 3). En los menores de 15 años, la proporción de muertes en la casa disminuyó entre los años 1990 y 2003, en el resto del país y en la Sex-ta Región $\left(R_{\text {resto }}=0,916, p<0,001 \mathrm{y}\right.$ $\left.\mathrm{R}_{\text {sexta }}=0,745, \mathrm{p}=0,002\right) \mathrm{y}$, consecuentemente,

Tabla 2. D istribución porcentual de las defunciones por lugar de ocurrencia en la Sexta Región y en el resto de C hile 1990-2003

\begin{tabular}{|ccccccc|}
\hline & \multicolumn{3}{c}{ Resto del país } & \multicolumn{3}{c|}{ Sexta Región } \\
& Hospital & Casa & Otro & Hospital & Casa & Otro \\
\hline 1990 & 45,8 & 45,9 & 8,2 & 44,7 & 46,0 & 9,3 \\
1991 & 45,1 & 46,7 & 8,2 & 43,0 & 47,2 & 9,9 \\
1992 & 45,0 & 46,9 & 8,1 & 45,6 & 44,7 & 9,7 \\
1993 & 44,5 & 47,2 & 8,3 & 45,0 & 45,5 & 9,5 \\
1994 & 44,7 & 47,1 & 8,2 & 45,4 & 45,1 & 9,5 \\
1995 & 45,2 & 46,4 & 8,4 & 46,3 & 44,8 & 8,8 \\
1996 & 45,7 & 46,2 & 8,1 & 46,3 & 44,6 & 9,1 \\
1997 & 45,8 & 46,0 & 8,2 & 47,4 & 43,4 & 9,2 \\
1998 & 45,9 & 46,1 & 7,9 & 48,5 & 42,4 & 9,1 \\
1999 & 46,0 & 46,3 & 7,7 & 47,6 & 43,7 & 8,8 \\
2000 & 45,9 & 45,8 & 8,3 & 48,0 & 42,1 & 9,9 \\
2001 & 45,5 & 45,8 & 8,7 & 47,3 & 42,8 & 9,9 \\
2002 & 40,2 & 47,0 & 12,7 & 43,0 & 43,2 & 13,8 \\
2003 & 42,2 & 48,9 & 8,9 & 45,0 & 45,7 & 9,3 \\
Total & 44,8 & 46,6 & 8,6 & 46,0 & 44,3 & 9,7 \\
\hline
\end{tabular}


aumentó el porcentaje de fallecimientos de niños en el hospital $\left(R_{\text {resto }}=0,77 ; p=0,001\right.$ y $R_{\text {sexta }}$ $=0,66 ; p=0,01$ ). Al comparar la proporción de decesos en el hospital, la Sexta Región tenía una menor proporción promedio que el resto del país $(69,89 \%$ vs $74,62 \%)$, diferencia estadísticamente significativa ( $p<0,0001$ ); en contraste con los fallecimientos en otro lugar, en que la región tuvo un promedio de $13,9 \%$ y el resto del país fue de $9,1 \%$ ( $p<0,0001$ ).

Defunciones en el grupo de adolescentes (Tabla 4). Las muertes de adolescentes en hospital mostraron una disminución en la Sexta Región y en el resto de Chile, que fue estadísticamente significativa para el resto del país $(R=0,635, p=0,015)$. Aumentaron las

Tabla 3. D istribución porcentual de las defunciones de los menores de 15 años según lugar de ocurrencia en la Sexta Región y en el resto de C hile 1990-2003

\begin{tabular}{|lcccccc|}
\hline & \multicolumn{3}{c}{ Resto del país } & \multicolumn{3}{c|}{ Sexta Región } \\
Año & Hospital & Casa & Otro & Hospital & Casa & Otro \\
\hline 1990 & 70,5 & 20,6 & 8,6 & 63,5 & 24,4 & 12,1 \\
1991 & 70,2 & 20,9 & 8,8 & 64,7 & 20,2 & 15,1 \\
1992 & 71,5 & 19,0 & 8,9 & 70,9 & 16,9 & 11,9 \\
1993 & 71,9 & 19,2 & 8,9 & 68,1 & 20,4 & 11,2 \\
1994 & 73,9 & 17,7 & 8,4 & 68,6 & 17,2 & 14,2 \\
1995 & 72,9 & 17,5 & 9,5 & 67,9 & 17,7 & 13,9 \\
1996 & 74,8 & 16,0 & 9,1 & 68,0 & 14,6 & 16,9 \\
1997 & 75,4 & 15,4 & 9,1 & 70,3 & 13,7 & 16,0 \\
1998 & 77,9 & 13,9 & 8,2 & 77,4 & 11,6 & 11,1 \\
1999 & 78,9 & 13,1 & 7,9 & 71,8 & 13,9 & 14,4 \\
2000 & 79,6 & 11,5 & 8,8 & 72,4 & 12,8 & 14,8 \\
2001 & 77,2 & 13,1 & 9,6 & 73,7 & 11,1 & 15,3 \\
2002 & 73,4 & 15,2 & 11,4 & 67,9 & 19,1 & 13,1 \\
2003 & 76,4 & 12,9 & 10,7 & 73,4 & 11,2 & 15,4 \\
Total & 74,1 & 16,9 & 9,0 & 69,5 & 16,6 & 13,9 \\
\hline
\end{tabular}

Tabla 4. D istribución porcentual de las defunciones de adolescentes según lugar de ocurrencia en la Sexta Región y resto de C hile 1990-2003

\begin{tabular}{|lcccccc|}
\hline & \multicolumn{3}{c}{ Resto del país } & \multicolumn{3}{c|}{ Sexta Región } \\
Año & Hospital & Casa & Otro & Hospital & Casa & Otro \\
\hline 1990 & 45,0 & 22,6 & 32,4 & 44,4 & 5,6 & 50,0 \\
1991 & 45,3 & 22,1 & 32,5 & 38,1 & 23,8 & 38,1 \\
1992 & 48,9 & 16,1 & 35,0 & 36,7 & 30,0 & 33,3 \\
1993 & 45,4 & 21,6 & 33,0 & 46,7 & 6,7 & 46,7 \\
1994 & 46,7 & 18,6 & 34,8 & 65,0 & 25,0 & 10,0 \\
1995 & 45,1 & 17,4 & 37,5 & 36,4 & 27,3 & 36,4 \\
1996 & 44,1 & 23,2 & 32,7 & 47,8 & 21,7 & 30,4 \\
1997 & 45,1 & 20,0 & 34,9 & 58,3 & 20,8 & 20,8 \\
1998 & 44,8 & 18,9 & 36,3 & 40,0 & 8,0 & 52,0 \\
1999 & 45,0 & 20,6 & 34,4 & 34,8 & 30,4 & 34,8 \\
2000 & 43,9 & 19,5 & 36,6 & 47,8 & 8,7 & 43,5 \\
2001 & 45,6 & 19,1 & 35,3 & 41,2 & 11,8 & 47,1 \\
2002 & 41,5 & 22,0 & 36,5 & 56,0 & 8,0 & 36,0 \\
2003 & 43,1 & 25,0 & 31,9 & 22,9 & 28,6 & 48,6 \\
Total & 45,0 & 20,4 & 34,5 & 43,2 & 18,1 & 38,7 \\
\hline
\end{tabular}


muertes en otro lugar, en los dos grupos geográficos aunque sin significación estadística. El promedio de muertes de adolescentes en otro lugar en el resto del país fue de $34,6 \%$, cifra que en los adultos alcanzó a 15,3\% ( $\mathrm{p}<0,0001)$.

Defunciones en el grupo de adultos 1990-2003 (Tabla 5). En este grupo etario, la evolución en el resto del país mostró una disminución de las muertes en hospital ( $R=0,61 ; p=0,02)$ y un incremento de los fallecimientos en otro lugar ( $R=0,56 ; p=0,04)$. En la Sexta Región no hubo variaciones significativas, pero, al comparar los promedios de muertes en hospital, éste era menor en la región (47,8\%) que en el resto de Chile (48,8\%), $(p=0,06)$.

Defunciones en el grupo de mayores de 65 años (Tabla 6). En el grupo de mayores de 65 años, se

Tabla 5. D efunciones de los adultos según lugar de ocurrencia en la Sexta Región y en el resto de C hile 1990-2003

\begin{tabular}{|lcccccc|}
\hline & \multicolumn{3}{c}{ Resto del país } & \multicolumn{3}{c|}{ Sexta Región } \\
Año & Hospital & Casa & Otro & Hospital & Casa & Otro \\
\hline 1990 & 50,4 & 35,3 & 14,3 & 48,4 & 33,1 & 18,5 \\
1991 & 49,5 & 35,6 & 14,9 & 44,8 & 34,4 & 20,8 \\
1992 & 49,6 & 35,7 & 14,7 & 47,8 & 31,7 & 20,5 \\
1993 & 48,3 & 36,3 & 15,3 & 46,8 & 33,9 & 19,3 \\
1994 & 48,8 & 36,4 & 14,8 & 47,4 & 32,7 & 19,9 \\
1995 & 49,1 & 35,7 & 15,2 & 48,6 & 33,9 & 17,5 \\
1996 & 49,7 & 35,4 & 14,9 & 47,3 & 33,5 & 19,3 \\
1997 & 49,7 & 34,9 & 15,5 & 51,1 & 31,0 & 17,9 \\
1998 & 49,5 & 35,4 & 15,1 & 49,7 & 32,1 & 18,3 \\
1999 & 49,6 & 35,7 & 14,7 & 48,4 & 32,6 & 19,0 \\
2000 & 49,0 & 35,3 & 15,7 & 48,1 & 32,9 & 19,0 \\
2001 & 48,8 & 35,3 & 16,0 & 47,1 & 33,1 & 19,9 \\
2002 & 44,1 & 37,6 & 18,3 & 47,1 & 33,2 & 19,7 \\
2003 & 46,5 & 38,6 & 14,9 & 46,5 & 35,6 & 17,9 \\
Total & 48,8 & 35,9 & 15,3 & 47,8 & 33,1 & 19,1 \\
\hline
\end{tabular}

Tabla 6. D istribución porcentual de las defunciones de mayores de 65 años según lugar de ocurrencia en la Sexta Región y resto de Chile 1990-2003

\begin{tabular}{|ccccccc|}
\hline & \multicolumn{3}{c}{ Resto del país } & \multicolumn{3}{c|}{ Sexta Región } \\
& Hospital & Casa & Otro & Hospital & Casa & Otro \\
\hline 1990 & 39,3 & 56,1 & 4,2 & 39,9 & 56,7 & 3,0 \\
1991 & 39,0 & 56,6 & 4,1 & 38,9 & 57,7 & 2,9 \\
1992 & 39,0 & 56,6 & 4,2 & 41,5 & 54,6 & 3,5 \\
1993 & 39,3 & 56,2 & 4,3 & 41,1 & 53,7 & 4,2 \\
1994 & 39,3 & 56,1 & 4,4 & 41,4 & 54,6 & 3,3 \\
1995 & 40,3 & 54,8 & 4,5 & 42,7 & 53,1 & 3,4 \\
1996 & 40,8 & 54,4 & 4,4 & 43,7 & 53,1 & 2,8 \\
1997 & 41,4 & 54,1 & 4,3 & 43,6 & 51,7 & 4,4 \\
1998 & 41,6 & 53,8 & 4,4 & 45,6 & 50,0 & 4,1 \\
1999 & 42,0 & 53,5 & 4,4 & 45,5 & 50,8 & 3,5 \\
2000 & 42,1 & 53,0 & 4,7 & 46,0 & 49,0 & 4,8 \\
2001 & 41,9 & 53,0 & 5,1 & 45,6 & 49,6 & 4,6 \\
2002 & 36,5 & 53,3 & 10,1 & 39,4 & 49,7 & 10,8 \\
2003 & 38,5 & 55,6 & 5,9 & 42,7 & 52,9 & 4,4 \\
Total & 40,2 & 54,8 & 5,0 & 43,0 & 52,7 & 4,3 \\
\hline
\end{tabular}


apreció una disminución de las muertes en la casa en la Sexta Región $(R=0,839 ; p=0,002)$ y en el resto del país $(\mathrm{R}=0,761 ; \mathrm{p}<0,0001)$. En contraste, hubo aumento de los fallecimientos en otro lugar $\left(R_{\text {sexta }}=0,573 ; p=0,03\right.$ y $\left.R_{\text {resto }}=0,574 ; p=0,03\right)$. Sin embargo, el porcentaje promedio de deceso en otro lugar fue bajo $(4,9 \%$ en las otras regiones y $4,3 \%$ en la región de O’Higgins).

La evolución del lugar de defunción por sexo y grupo etario en Chile, reveló que los hombres presentaron un aumento significativo de muerte en la casa en el grupo de adultos ( $R=0,54, p=0,04)$ y en otro lugar en los mayores de 65 años $(R=0,57$, $p$ $=0,03$ ), en oposición a un descenso de muertes en hospital en los adultos $(R=0,61, p=0,02)$ y en la casa en los senescentes ( $R=0,68, p=0,007$ ).

En las mujeres, existió un aumento de la muerte en otro lugar para las personas adultas $(R=0,55, p$ $=0,04)$ y senescentes ( $R=0,58, p=0,03)$ y un descenso significativo de las defunciones en la casa en el grupo mayor de 65 años ( $R=0,86, p<0,0001$ ).

En el grupo infantil, ambos sexos mostraron un descenso significativo de las muertes en la casa $\left(R_{\text {niños }}=0,9, p<0,001 ; R_{\text {niñas }}=0,9, p<0,001\right)$ y un aumento significativo de los decesos en hospital $\left(R_{\text {niños }}=0,73, p=0,003 ; R_{\text {niñas }}=0,81, p<0,001\right)$ (Figura 1).
En el caso de los adolescentes, se observó un descenso significativo de las muertes en el hospital en los hombres ( $R=0,53 ; p=0,05$ ) y un aumento significativo de los decesos en otro lugar en las mujeres ( $R=0,597 ; p=0,02)$, aunque la proporción de muertes en otro lugar fue significativamente mayor para los varones, lo que atribuimos a que su proporción de muertes por traumatismos y envenenamientos es significativamente mayor, rRiesgo relativo masculino $=1,54$ $(1,46-1,62)$, con $p>0,000$.

Al considerar los grupos de enfermedades que causan la muerte y el lugar de defunción, destaca el grupo de enfermedades del aparato circulatorio, en que la evolución mostró un descenso significativo de las defunciones en la casa ( $R=0,88, p<0,0001$ ) y los tumores que revelan un incremento de la proporción de decesos en la casa $(R=0,63, p=0,01)$ (Figuras 2 y 3).

Atención médica en la última enfermedad (Tabla 7). En el país se ha observado un aumento significativo de la atención médica ( $R=0,985, p<0,0001)$, lo que es similar a lo acontecido en la Sexta Región $(\mathrm{R}=0,957$, $\mathrm{p}$ $<0,0001)$. La proporción de muertes con atención médica de la última enfermedad varió de

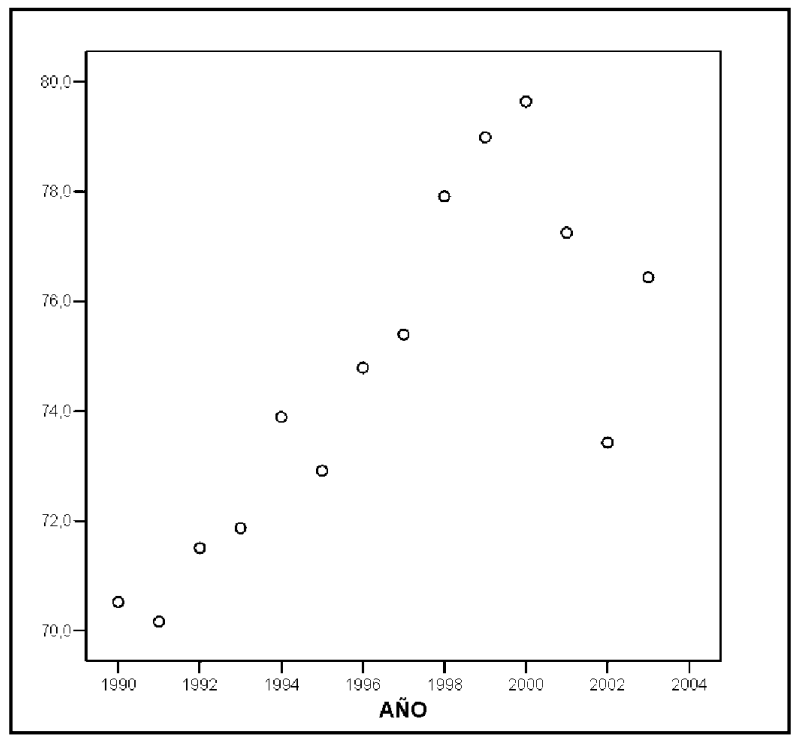

Figura 1. Defunciones de menores de 15 años en hospital 1990-2003. 


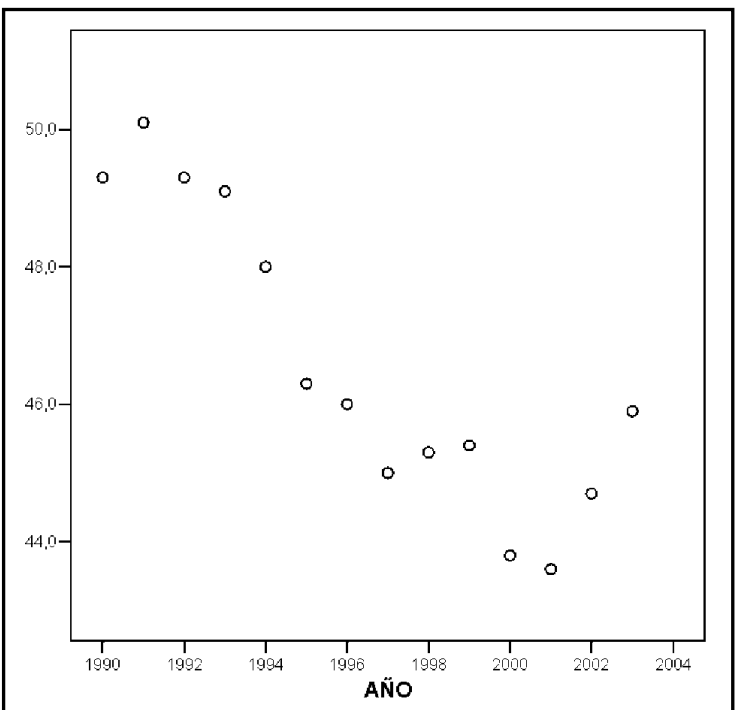

Figura 2. Defunciones por enfermedades del aparato circulatorio en la casa 1990-2003.

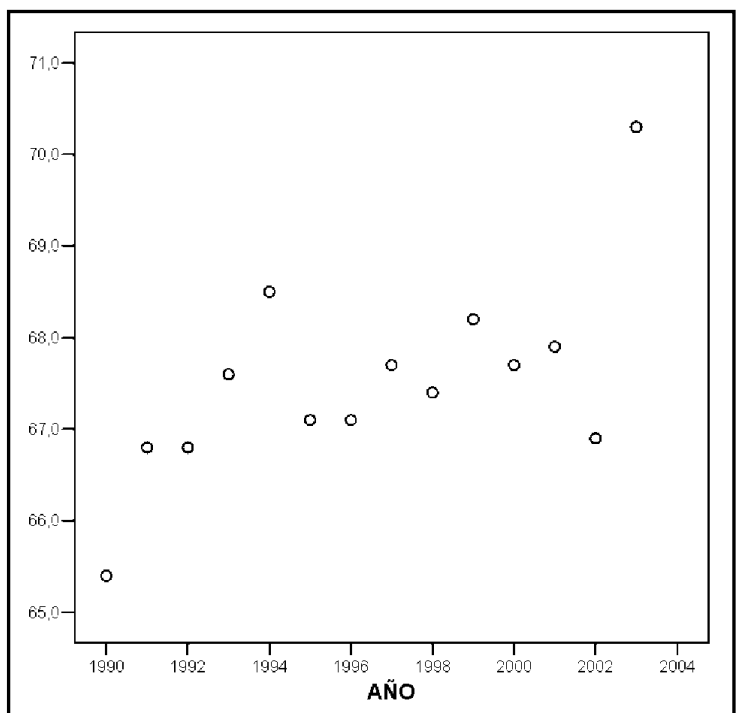

Figura 3. Defunciones por tumores en la casa 19902003.

Tabla 7. D istribución porcentual de las defunciones según atención médica en la última enfermedad en el resto del país y la Sexta Región. 1990-2003

\begin{tabular}{|lcccccc|}
\hline & \multicolumn{3}{c}{ Resto del país } & \multicolumn{3}{c|}{ Sexta Región } \\
Año & Con atención & Sin atención & Ignorado & Con atención & Sin atención & Ignorado \\
\hline 1990 & 74,7 & 17,8 & 7,7 & 71,1 & 15,6 & 9,3 \\
1991 & 76,2 & 16,6 & 7,4 & 74,4 & 15,6 & 9,8 \\
1992 & 77,5 & 18,2 & 4,4 & 75,8 & 16,3 & 9,6 \\
1993 & 77,3 & 19,4 & 3,4 & 75,4 & 18,0 & 9,5 \\
1994 & 78,5 & 19,2 & 2,5 & 76,0 & 18,7 & 9,5 \\
1995 & 78,4 & 17,3 & 4,6 & 79,3 & 16,1 & 8,8 \\
1996 & 79,8 & 13,9 & 6,6 & 79,9 & 15,1 & 9,1 \\
1997 & 80,2 & 11,7 & 8,2 & 81,3 & 11,8 & 9,2 \\
1998 & 80,7 & 10,8 & 8,6 & 82,4 & 12,3 & 9,1 \\
1999 & 81,4 & 10,2 & 8,4 & 82,7 & 12,2 & 8,8 \\
2000 & 81,4 & 10,5 & 8,2 & 82,2 & 12,9 & 9,9 \\
2001 & 81,7 & 9,6 & 8,7 & 83,4 & 12,6 & 9,9 \\
2002 & 83,2 & 8,3 & 8,5 & 83,4 & 10,5 & 13,8 \\
2003 & 83,9 & 7,5 & 8,5 & 84,2 & 12,0 & 9,3 \\
\hline
\end{tabular}

$74,7 \%$ en 1990, para el resto del país y $71,1 \%$ en la Sexta Región en el mismo año, hasta 83,95\% de defunciones con atención el año 2003 en el resto de Chile y $84,2 \%$ en la Sexta Región, ese año.
$\mathrm{Al}$ analizar la proporción de atención médica por grupo etario, se apreció un aumento de la atención en todos los grupos, excepto el caso de los adolescentes en el resto del país, destacando el grupo infantil en el que se apreció un incremento 
de defunciones con atención en la Sexta Región y en el resto del país, con un rango que va desde $70,4 \%$ de las defunciones con atención médica el año 1990 en el resto de Chile, a 77,2\% con atención el año 2003. En el caso de la Sexta Región, el rango se extendió desde 66,7\% con atención en 1990 a 76,1\% en el año 2003.

La comparación de la proporción de muertes con atención médica en el grupo infantil con la proporción de adultos y senescentes, reveló que el grupo infantil tiene una situación privilegiada, con una diferencia estadísticamente significativa. $(\mathrm{t}=10,04, \mathrm{p}<0,0001)$. El grupo de adolescentes tuvo las proporciones menores de atención médica $(39,3 \%$ para el resto del país y $45,5 \%$ en la Sexta Región) y una situación desmedrada en comparación con el resto de los grupos, con diferencias estadísticamente significativas.

\section{DiscUSIÓN}

Nuestros resultados permiten afirmar que la cobertura de la atención médica en la última enfermedad aumentó, en la región y en el resto del país, considerándose un indicador de mejoramiento del sistema de salud.

El análisis del lugar de la defunción, mostró diferencias según la enfermedad causal de la muerte, el grupo etario estudiado y la variable geográfica.

Los fallecimientos en la casa de enfermos del aparato circulatorio disminuyeron, en contraste con el aumento de los decesos en el domicilio de los pacientes con tumores, lo que traduciría una decisión del paciente y su familia.

En la edad infantil, la realidad es categórica: tanto en nuestra región como en el resto de Chile, ha existido una evolución decreciente de las muertes en domicilio, resultado muy favorable de las políticas de salud pública que han propiciado programas que permiten una buena cobertura de la atención de salud, en la atención primaria y en el nivel de mayor complejidad. Además, los resultados apoyan la evaluación favorable de la promoción y prevención que han permitido educar, especialmente a las madres, en el reconocimiento precoz de los signos y síntomas que indican gravedad $\mathrm{y}$, por tanto, necesidad de acudir a un centro de salud.

La situación en los adolescentes es especial, ya que si bien los decesos en esta edad son menores, la causalidad fue en más de la mitad de los casos atribuible a traumatismos y envenenamientos, que producen la muerte en la casa o en otros lugares distintos del hospital. El enfrentamiento, entonces, pasa por un enfoque social que considere el manejo de la violencia y de la depresión en este grupo etario.

Los resultados en los adultos no fueron concluyentes respecto a las variaciones encontradas. Sin embargo, podemos mencionar el desafío para el sistema de salud de la Sexta Región, debido a que la proporción de fallecimientos en el hospital fue menor que en el resto del país.

El lugar de deceso de los mayores de 65 años mostró aumento de las muertes en otro lugar. Pero, la proporción de estas muertes es baja aún y la tendencia pareciera seguir lo que señala la literatura, en el sentido de que los adultos mayores están decidiendo pasar sus últimos días en lugares distintos a sus casas $u$ hospitales ${ }^{11}$. No obstante lo anteriormente señalado, podría plantearse que los ancianos no han tenido acceso al sistema hospitalario y por eso aumentan las muertes en otros lugares. A la luz de nuestros resultados, esta hipótesis no tiene base, pues la proporción de muertes en los hospitales también aumentó.

Existen consideraciones éticas referentes al lugar en que las personas «deben morir», lo que evidentemente está condicionado por la edad del paciente. En Chile, sólo 1,2\% de la población censada son padres de los jefes de hogar, lo que podría indicar que no parece cercana la posibilidad de programas de cuidados paliativos de enfermos añosos o terminales, en sus hogares, como alternativa para permitir que la muerte acontezca en el hogar, en un ambiente de cariño especialmente necesario a la hora de morir. 


\section{REFERENCIAS}

1. WHO. The World Health Report 2005. Annex Table 1 Basic indicators for all WHO Member States 174-181.

2. Kortchagina I, Ovtcharova L, Prokofieva L, Festy P, Verger D. Conditions de vie et pauvreté en Russie. Ëconomie et Statistique № 383-384-385, 2005.

3. Desesquelues A, Richet-Mastain L. Bilan démographique 2003: Stabilité des naissances, augmentation de dècés. Insee Premiere № 948 Février 2004. Disponible en www.insee.fr (Consultado el 26 de julio de 2006).

4. China 2005. Atención médica y protección sanitaria. Disponible en: www.china.org.cn (Consultado el 26 de julio de 2006).

5. Mejer L, Robert-Bobée I. Mortalité des femmes et environment familial. Rôle protecteur de la vie de famille. Insee Premiere № 892 Avril 2003. Disponible en: www.insee.fr (Consultado el 26 de julio de 2006).

6. Montell C, Robert-Bobée I. Les differences sociales de mortalité: en augmentation chez les homes, stables chez les femmes. Insee Premiere № 1025 Juin 2005. Disponible en: www.insee.fr (Consultado el 26 de julio de 2006).

7. LeIVA H, LEón F. Evolución de la mortalidad en Chile 1990-2003. Rev Soc Med VI Reg 2006; 3: 711-20.

8. Medina E, KaEMPFer A. Mortalidad del Adulto en Chile. Rev Méd Chile 2000; 128: 1144-9.

9. Szот J. Mortalidad por infarto agudo al miocardio en Chile: 1990-2001. Rev Méd Chile 2004; 132: 1227-33.

10. RoSELO M, GuZMÁN S. Mortalidad por infarto agudo de miocardio: distribución geográfica y lugar de ocumencia. Costa Rica, 1970-2002. Rev Costarric Cardiol 2003; 5: 25-29. Disponible en: www.scielo.sa.cr (Consultado el 26 de julio de 2006).

11. MeIJer I, Fichter M, Schröppel H. Riesgo de mortalidad en octogenarios y nonagenarios en relación con síntomas psíquicos, causa y lugar de fallecimiento. Resultados longitudinales de un estudio epidemiológico de seguimiento en una muestra comunitaria. Eur J Psychiat (Ed. Esp) 2004; 18: 4561. Disponible en www.scielo.isciii.es. (Consultado el 26 de julio de 2006).

12. GuPTA I, SanKar D. Medical Attention at Death: Evidence from India Institute of Economic Grow Delhi Discussion Papers № 45 Disponible en: http://ieg.nic.in/dis_ind_45.pdf (Consultado el 26 de julio de 2006).

13. Kaufman S. And a Time to Die: How American Hospitals Shape the End of Life. The University of Chicago Press 2005; 1-8. Disponible en: www.press.uchicago.edu/Misc/Chicago/ 426858.html (Consultado el 26 de julio de 2006).

14. Johnson K, Kuchibhatala M, Sloane R, Tanis D, Galanos A, Tulsky J. Ethnic Differences in the Place of Death of Elderly Hospice Enrollees. J Am Geriatr Soc 2005; 53: 2209-15. Disponible en: www.blackwell-synergy.com (Consultado el 7 de agosto de 2006).

15. Dureux-Brouiшaud M. Problèmes éthiques posés par la mise en place d'une équipe mobile gérontologique de soins palliatifs. DEA 1993. Disponible en http://infodoc.inserm.fr/ethique/ (Consultado el 26 de julio de 1006).

16. Nagaraja J, Menkedick J, Phelan K, Ashiey P, Zhang $\mathrm{X}$, Lanphear B. Deaths From Residencial Injuries in US Children and Adolescents, 1985-1997. Pediatrics 2005; 116; 454-61.

17. Cels A, Gómez-Lomel Z, Armas J. Tendencias de mortalidad por traumatismos y envenenamientos en adolescentes. México, 1979-1997. Salud Pública Méx 2003, vol. 45 suppl. 1, ps8-s15. ISSN 00363634.

18. Gran M, Torres R, Martínez M, Viera R. Mortalidad general y por causas externas en adolescentes cubanos. 2003 Disponible en www.sld.cu/galerias/pdf/sitios/dne/ Consultado el 30 de noviembre de 2006). 